\title{
ERROR EXPRESSIONS FOR CERTAIN CONTINUED FRACTIONS*
}

\author{
BY RAYMOND GARVER
}

Every discussion of the subject shows that the error committed in taking the $n$th convergent $p_{n} / q_{n}$ as the true value of the simple continued fraction

$$
a_{1}+\frac{1}{a_{2}}+\frac{1}{a_{3}}+\cdots+\frac{1}{a_{n}}+\ldots, \quad\left(a_{i} \text { positive integers }\right)
$$

is less than $1 /\left(q_{n} q_{n+1}\right)$, and greater than $a_{n+2} /\left(q_{n} q_{n+2}\right)$ in absolute value. Further, $p_{n} / q_{n}$ is smaller than the true value if $n$ is odd, and larger if $n$ is even, provided the continued fraction does not terminate.

The purpose of the present paper is to supply alternate error limits for the important case when the continued fraction is the expansion of the square root of an integer $N$. These limits have the advantage that they do not require the computation of any convergent beyond the $n$ th. Further, when applied as corrections to the $n$th convergent, they practically always lead to a much closer approximation than do the general error limits. Our results may be summarized as follows:

Given $N^{1 / 2}$ expressed in the form (1), with $n$th convergent $p_{n} / q_{n}$, and with $k$ defined $\dagger$ as $\left|p_{n}^{2}-N q_{n}^{2}\right|$. If $n$ is even, the error committed in taking $p_{n} / q_{n}$ as the value of $N^{1 / 2}$ is greater than $k /\left(2 p_{n} q_{n}\right)$, and less than $k /\left(2 q_{n}^{2} N\right)^{1 / 2}$, in absolute value. If $n$ is odd, this statement is true with "greater than" and "less

* Presented to the Society, August 31, 1932.

$\dagger$ We might affix a subscript to $k$, but it does not seem necessary. If $p_{n}$ and $q_{n}$ are large the arithmetic required to compute $k$ might be considerable if it were necessary to multiply out $p_{n}^{2}-N q_{n}^{2}$. This is not, however, usually necessary. For, as is well known, the values that $k$ can take on come in cycles, and the complete cycle can be formed easily from small values of $n$ in many cases. It is also known that $k$ is less than $2 N^{1 / 2}$, which will render the complete squaring of $p_{n}$ and $q_{n}$ unnecessary even when the complete cycle of values has not been formed. Finally, if $n=m c$, where $m$ is a positive integer and $c$ is the number of partial quotients in the period of the continued fraction expansion of $N^{1 / 2}$, then $k$ is 1 . This case will receive special mention at the end of the present paper. 
than" interchanged. These limits are ordinarily more satisfactory than the limits $1 /\left(q_{n} q_{n+1}\right)$ and $a_{n+2} /\left(q_{n} q_{n+2}\right)$.

To obtain the desired error expressions we have merely to write

$$
E \equiv\left|p_{n} / q_{n}-N^{1 / 2}\right|=k /\left(q_{n}\left(p_{n}+N^{1 / 2} q_{n}\right)\right) .
$$

Now if $n$ is even, and we replace $N^{1 / 2}$ by $p_{n} / q_{n}$, which is larger than it, we have at once $E>k /\left(2 p_{n} q_{n}\right)$. And if we, instead, replace $p_{n}$ by $N^{1 / 2} q_{n}$, which is smaller than it, we find $E<k /\left(2 q_{n}^{2} N^{1 / 2}\right)$. To render this upper limit convenient for computation we may now replace $N^{1 / 2}$ by any approximation which is too small, say $p_{n-1} / q_{n-1}$ or the better approximation $N q_{n} / p_{n}$. If the $(n+1)$ st convergent can be found easily it will be still better. The reader will see at once the modifications which must be made in these statements when $n$ is odd.

It seems desirable to compare these error expressions with the general limits $1 /\left(q_{n} q_{n+1}\right)$ and $a_{n+2} /\left(q_{n} q_{n+2}\right)$. First, the upper limit just determined is always less than $1 /\left(q_{n} q_{n+1}\right)$, when $n$ is even. To see this note that the inequality

$$
\frac{k}{q_{n}\left(p_{n}+N^{1 / 2} q_{n}\right)}<\frac{1}{q_{n} q_{n+1}}
$$

implies that

$$
k q_{n+1}<p_{n}+N^{1 / 2} q_{n}<2 p_{n}-\frac{a_{n+2}}{q_{n+2}} .
$$

Since $k q_{n+1}$ is an integer and $a_{n+2}<q_{n+2}$, we then have $k q_{n+1}$ $\leqq 2 p_{n}-1$. Now $k /\left(2 q_{n}^{2} N^{1 / 2}\right)$ is less than $1 /\left(q_{n} q_{n+1}\right)$ provided $k q_{n+1}<2 N^{1 / 2} q_{n}$; and this inequality will be established as soon as we show that $2 p_{n}-1<2 N^{1 / 2} q_{n}$. This last is easily seen to be reducible to $E<1 /\left(2 q_{n}\right)$, which is satisfied since $E$ is certainly less than $1 /\left(q_{n} q_{n+1}\right)$, and $q_{n+1} \geqq 2$ when $n$ is even.

We have mentioned that $N^{1 / 2}$ may be replaced by suitable approximations to give a more convenient, though poorer, upper limit. Suppose we use $p_{n-1} / q_{n-1}$. A sufficient condition that the new upper limit still be less than $1 /\left(q_{n} q_{n+1}\right)$ is that $2 p_{n}-1$ be less than $2 q_{n} p_{n-1} / q_{n-1}$. Using the relation $p_{n} q_{n-1}-q_{n} p_{n-1}$ $=(-1)^{n}$ this reduces to $2<q_{n-1}$. Hence the modified upper limit is less than $1 /\left(q_{n} q_{n+1}\right)$ unless $q_{n-1}$ is 1 or 2 ; it may even be less in these trivial cases. The same statement holds, a fortiori, when 
the modified upper limit is formed with a better approximation than $p_{n-1} / q_{n-1}$.

The lower limit $k /\left(2 p_{n} q_{n}\right)$ for $n$ even is greater than or equal to $a_{n+2} /\left(q_{n} q_{n+2}\right)$ provided $a_{n+2} \leqq q_{n+1}$. This sufficient condition will certainly be satisfied unless $n$ is small, for it is known that $a_{n+2}$ is less than $2 N^{1 / 2}$. To establish this relation between the lower limits note that the inequality

implies that

$$
\frac{k}{q_{n}\left(p_{n}+N^{1 / 2} q_{n}\right)}>\frac{a_{n+2}}{q_{n} q_{n+2}}
$$

$$
k q_{n+2}>a_{n+2}\left(2 p_{n}-E q_{n}\right) .
$$

Now $k /\left(2 p_{n} q_{n}\right) \geqq a_{n+2} /\left(q_{n} q_{n+2}\right)$ provided $k q_{n+2} \geqq 2 p_{n} a_{n+2}$, and it follows from (5) that this condition will be satisfied whenever $a_{n+2} E q_{n} \leqq 1$. But since $E<1 /\left(q_{n} q_{n+1}\right)$, the sufficient condition as stated follows.

It is not easy to set up a simple example in which the new lower limit is poorer than the old. One can be found in connection with

(6) $31^{1 / 2}=5+\frac{1}{1}+\frac{1}{1}+\frac{1}{3}+\frac{1}{5}+\frac{1}{3}+\frac{1}{1}+\frac{1}{1}+\frac{1}{10}+\cdots$

If the second convergent, 6 , be taken as the value of $31^{1 / 2}$, the error is greater than $5 / 12$ by the expression $k /\left(2 p_{n} q_{n}\right)$, and greater than $3 / 7$ by the expression $a_{n+2} /\left(q_{n} q_{n+2}\right)$. In this case, of course, we have $a_{n+2}>q_{n+1}$. If the fourth convergent be used instead of the second the two lower limits give the same value, but our new upper limit is better for the sixth convergent.

We turn to the consideration of the case where $n$ is odd. An odd convergent is less in value than the continued fraction, so we have $p_{n} / q_{n}=N^{1 / 2}-E$, and in place of (3) we find

$$
k q_{n+1}<2 p_{n}+E q_{n}<2 p_{n}+\frac{1}{q_{n+1}} .
$$

It then follows that $k q_{n+1} \leqq 2 p_{n}$. And this is exactly the condition necessary to insure that the new upper limit $k /\left(2 p_{n} q_{n}\right)$ is not greater than $1 /\left(q_{n} q_{n+1}\right)$.

When $n$ is odd (5) must be written 


$$
k q_{n+2}>a_{n+2}\left(2 p_{n}+E q_{n}\right) \text {, }
$$

from which it follows that $k q_{n+2} \geqq 2 p_{n} a_{n+2}+1$. The lower limit for the case $n$ odd, that is, $k /\left(2 q_{n}^{2} N^{1 / 2}\right)$, will obviously be greater than $a_{n+2} /\left(q_{n} q_{n+2}\right)$ provided $2 p_{n} a_{n+2}+1>2 a_{n+2} q_{n} N^{1 / 2}$. And a moment's calculation, not greatly different from those already used, shows that this last inequality is certainly satisfied unless $2 a_{n+2}>q_{n+1}$. This is again an exceptional condition, which can arise only for small values of $n$. Thus in $2^{1 / 2}=1+\frac{1}{2}+\frac{1}{2}+\cdots$, if we take the first convergent 1 as the value, we find the error greater than $1 / 2^{3 / 2}$ by the formula $k /\left(2 q_{n}^{2} N^{1 / 2}\right)$, and greater than $2 / 5$ by the formula $a_{n+2} /\left(q_{n} q_{n+2}\right)$. In this example the second lower limit is better than the first. But this is no longer true when $n$ is 3 or larger.

It is again true that $N^{1 / 2}$ must be replaced by a rational approximation to get a lower limit suitable for computation; this time the approximation must be too large to insure that we still have a lower limit, but the values $p_{n-1} / q_{n-1}, N q_{n} / p_{n}$, $p_{n+1} / q_{n+1}$, now have this property. And it is easy to show that the limit obtained with the aid of the first of these is larger than $a_{n+2} /\left(q_{n} q_{n+2}\right)$ whenever $q_{n-1}>2 a_{n+2}$.

These general proofs that the new limits can only in exceptional cases be poorer than the old limits do not begin to show the advantage possessed by the new limits in most calculations. This can better be pointed out by taking the difference of the upper and lower limits in the two cases. If we replace $N^{1 / 2}$ in the one limit by the always available approximation $N q_{n} / p_{n}$, we find that the difference of our two limits is $k^{2} /\left(2 p_{n} q_{n}^{3} N\right)$. This difference allows us to estimate at once how accurately we can approximate with the aid of the $n$th convergent. The corresponding difference between the usual limits $1 /\left(q_{n} q_{n+1}\right)$ and $a_{n+2} /\left(q_{n} q_{n+2}\right)$ is $1 /\left(q_{n+1} q_{n+2}\right)$. The ratio of the first difference to the second is $R=k^{2} q_{n+1} q_{n+2} /\left(2 p_{n} q_{n}^{3} N\right)$. Making obvious substitutions for $q_{n+1}$ and $q_{n+2}$ in terms of the earlier $q$ 's, and remembering that $k$, as well as each $a_{i}$, is less than $2 N^{1 / 2}$, we find $R<2\left(2 N^{1 / 2}+1\right)^{3} /\left(p_{n} q_{n}\right)$. The ratio $R$ is then certainly less than 1 except for small values of $p_{n}$ and $q_{n}$. In fact, $R$ clearly approaches zero as $n$ increases.

To have one numerical example, consider the approximation of $2^{1 / 2}$ by its sixth convergent, $99 / 70=1.414285714+$. The old limits simply show that this approximation is too large by an 
amount larger than 0.0000700 and smaller than 0.0000846 . However the new limits, with $N^{1 / 2}$ in the upper limit replaced by $140 / 99$, tell us that the error is larger than 0.000072150 and smaller than 0.000072158 . With this tremendous gain in accuracy we can at once say definitely that $2^{1 / 2}=1.41421356$, correct to 8 decimal places.

The limits of the present paper are applicable, under a certain restriction, to a well known method of approximating square roots by iteration. If $a_{1}$ is a first approximation to $N^{1 / 2}$, a second and closer approximation is obtained by taking $a_{2}=\left(a_{1}+N / a_{1}\right) / 2$, a third approximation is formed similarly from the second, and so on. This method has been known since ancient times and recently has been dealt with in a number of papers. ${ }^{*}$ Nevertheless it is interesting to point out that if $c$ and $m$ have the significance of the second footnote of this paper, and if $a_{1}$ is taken as $p_{m c} / q_{m c}$, that is, the $m c$ th convergent to $N^{1 / 2}$, then $a_{2}$ is a later convergent, in fact, the $2 m c$ th. $\dagger$ Then $a_{3}$ is the $4 m c$ th convergent, and so on. There is, of course, ample justification for using the continued fraction expansion of $N^{1 / 2}$ to obtain $a_{1}$; we naturally want a close approximation without taking an unduly cumbersome fraction, and this is exactly what we obtain by using a convergent. And if we take the additional trouble to compute $p_{c} / q_{c}$, taking $m=1$ for convenience, we now know that the iterative method continues to give convergents of the form $p_{m c} / q_{m c}$. Our limits then apply, with $k=1$, and with $n$ even, provided we form at least $a_{2}$. It is worthwhile mentioning that the lower limit $1 /\left(2 p_{n} q_{n}\right)$, if we write $a_{i}=p_{n} / q_{n}$, is equal to Bouton's lower limit $\left(a_{i-1}-a_{i}\right)^{2} /\left(2 a_{i}\right)$, and is much easier to use. The proof of the equality is not difficult to make.

Similarly, the upper limit $1 /\left(2 q_{n}^{2} N^{1 / 2}\right)$ is equal to Bouton's upper limit $a_{i}\left(a_{i-1}-a_{i}\right)^{2} /(2 N)$ in case $N^{1 / 2}$ is replaced by $N q_{n} / p_{n}$. Of course if a better approximation is available a better upper limit than Bouton's can be obtained.

The University of California at Los Angeles

* Bouton, Annals of Mathematics, (2), vol. 10 (1908-9), pp. 167-72; James, American Mathematical Monthly, vol. 31 (1924), pp. 471-5.

$\dagger$ This follows from a theorem which may be found in Chrystal's Algebra, Part II, 1889, pp. 440-441. In a note appearing in the American Mathematical Monthly, vol. 39 (1932), pp. 533-535, I have gone into this point in more detail. 